\title{
Filigrane
}

Écoutes psychothérapiques

\section{Du roc du biologique au futur des civilisations. Entretien avec Essedik Jeddi}

\section{Michel Peterson}

Volume 21, numéro 1, printemps 2012

URI : https://id.erudit.org/iderudit/1012883ar

DOI : https://doi.org/10.7202/1012883ar

Aller au sommaire du numéro

Éditeur(s)

Revue Santé mentale au Québec

ISSN

1192-1412 (imprimé)

1911-4656 (numérique)

Découvrir la revue

Citer ce document

Peterson, M. (2012). Du roc du biologique au futur des civilisations. Entretien avec Essedik Jeddi. Filigrane, 21(1), 91-116. https://doi.org/10.7202/1012883ar d'utilisation que vous pouvez consulter en ligne. 


\title{
Du roc du biologique au futur des civilisations. Entretien avec Essedik Jeddi
}

\author{
Michel Peterson
}

MP : Essedik Jeddi, vous êtes de passage à Montréal. Vous avez fait plusieurs séjours au Québec et cette fois-ci vous y êtes pour une série d'interventions à la Société psychanalytique de Montréal et au Département de psychologie de l'Université du Québec à Montréal. Je voudrais en profiter pour faire avec vous le tour de votre parcours, comprendre ce qui vous a conduit ici parmi d'autres endroits.

Vous êtes né le 7 août 1940 à Monastir en Tunisie. Vous êtes marié, vous êtes père de trois enfants et vous avez trois petits-enfants. Vous avez beaucoup voyagé. Vous vous êtes engagé dans plusieurs causes. Vous avez fait une partie de vos études en Tunisie, puis à la Faculté de médecine de Lyon, en France, dans les années 68 . Vous êtes donc médecin, physiologiste, neuropsychiatre, psychanalyste et, comme si cela ne suffisait pas, traducteur et réalisateur de cinéma. Vous avez d'ailleurs écrit trois films. Voilà un parcours richissime et complexe. J'aimerais dans un premier temps que vous nous disiez comment vous en êtes venu à la médecine et à la physiologie.

EJ : Tout d'abord, effectivement, je suis dans ma soixante-douzième année. Je suis issu d'une famille d'enseignants, de ce que nous appelons, nous, des notables de la plume ('âylat al-qalam, composée de notaires, de jurisconsultes, de juges, d'enseignants, etc.), par opposition aux familles du makhzin, c'est-àdire les notables du pouvoir qui exercent des tâches allant des plus simples aux plus hautes.

Je suis le neuvième d'une famille de dix enfants, mais mon épouse ne pouvait accepter d'avoir neuf enfants; il m'était donc impossible de reproduire dans ma descendance ma propre position de neuvième enfant! (rire) Finalement, nous avons convenu de n'en faire que trois, une fille (Latifa) et deux garçons (Mohamed-Ali et Mourad-Mokhtar). Lors de mon premier long séjour au Canada, Mohamed-Ali et Mourad-Mokhtar devaient avoir 
respectivement 6 et 5 ans. Le Canada, et particulièrement Montréal, occupait une place importante dans le discours de ma famille, surtout que nous recevions assez souvent un certain nombre de collègues universitaires de Montréal qui participaient alors aux différents symposia que j'avais eu à organiser. Cette place qu'occupait le Canada et l'émerveillement qu'évoquait Montréal ne pouvaient qu'influencer mes deux garçons; c'est peut-être ce qui les a poussés à faire leur troisième cycle d'études supérieures à Toronto et à Montréal. L'un d'eux, Mohamed-Ali, est actuellement canadien, ce qui ne peut que renforcer davantage mes liens avec votre pays. J'ajouterais de plus que je suis grand-père de trois filles merveilleuses: Aïcha, Sélima et Sarra.

MP : Dans quelles circonstances êtes-vous venu au Québec pour la première fois?

Je suis venu au Québec pour la première fois en 1982. J'avais été invité par le professeur H.B.M. Murphy, que j'avais eu l'honneur de rencontrer à Dakar, lors d'un colloque organisé à la mémoire de mon ami le professeur Henri Collomb. J'y avais également rencontré le professeur Gilles Bibeau, qui avec le $\mathrm{D}^{\mathrm{r}}$ Murphy et la professeure Ellen Corin, m’avait invité à Montréal. Une trame d'échanges dynamiques s'était alors tissée entre le Service de psychiatrie universitaire que je dirigeais à l'hôpital Razi à Tunis, d'une part, et le Département de psychiatrie transculturelle de l'Université McGill, que dirigeait le professeur H.B.M. Murphy, ainsi qu'avec le GIRAME $^{1}$ et le Département d'anthropologie et de recherche en anthropologie médicale et psychiatrique, que dirigeait le professeur Gilles Bibeau. Le professeur Murphy avait eu à co-animer successivement dans mon unité de recherche, à Tunis, deux séminaires. L'un portait sur l'élaboration en équipe d'une fiche épidémiologique et le second, sur une première évaluation de cette fiche. De ce travail, et à partir des rencontres répétées à Tunis et à Montréal, avait émergé le projet d'un Master en psychiatrie sociale et transculturelle associant l'Université McGill (le Département de psychiatrie transculturelle) et la Faculté de médecine de Tunis (l'unité de recherche et de soins en psychiatrie sociale et transculturelle que je dirigeais à l'hôpital Razi). C'est seulement après le décès du professeur Murphy que ce projet a pu être finalisé avec le professeur Ray Prince, son successeur au Département. Il a été présenté aux conseils respectifs des universités ainsi qu'auprès de l'OMS pour accord et financement. Ce projet de Master devait être ouvert aux médecins psychiatres de tous les pays africains francophones. La formation théorique et pratique devait se faire une année à l'Université McGill et une deuxième année à l'Université de Tunis. Le professeur Prince avait visité à deux reprises la 
Tunisie pour étudier ce projet et conclure à sa faisabilité. Malheureusement, ce projet n'a pu voir le jour, mais son existence dans les archives témoigne de la nature et de la solidité des liens et des échanges que j'ai, et que nous avons eus, avec les universités de Montréal et le Québec. Depuis, nos cercles d'échanges scientifiques et nos liens d'amitié avec Montréal et le Québec se sont encore enrichis et étendus, puisque j'ai l'honneur et le plaisir aujourd'hui d'être parmi vous et de mériter de partager votre vie familiale.

MP : Bien. Une fois mis en place ces premiers éléments biographiques et professionnels qui ont toujours, quoi qu'on en dise, leur importance dans la filiation, dans la transmission, dans notre manière d'être sujet dans le monde, j'aimerais que nous nous penchions sur votre richissime parcours théoricoclinique. Vous avez côtoyé une multiplicité de grands chercheurs, de grandes figures et parmi elles, Gisela Pankow, qui a compté beaucoup dans votre parcours.

J'aimerais d'abord que nous parlions d'un concept que vous avez développé très tôt, entre 1969 et 1977, et que vous avez appelé le facteur thermotactile. Il concerne la thermorégulation comportementale et met en question le rapport entre la peau et la bouche. Nous avons l'habitude de dire que la peau est une extension de l'oralité, mais vous voyez les choses d'une façon singulièrement différente et selon vous, ce n'est pas l'oralité qui intervient en premier dans la constitution du psychisme, mais plutôt la régulation thermotactile. En 69 - il est important de le mentionner -, vous aviez adressé votre premier article de fond sur la question à Bowlby. Il ne vous a pas répondu, mais vous avez eu la surprise un peu plus tard de vous rendre compte qu'il avait introduit ce concept dans sa théorie de l'attachement. De quoi s'agit-il avec ce facteur thermotactile et par quel biais cela vous a-t-il introduit à d'autres travaux qui, à mon sens, ont à voir avec votre conception du traitement des psychoses?

EJ : Avant de répondre directement à cette question, permettez-moi de situer le début de mon travail sur le facteur thermotactile. En fait, j'avais commencé ma carrière parallèlement à ma formation en médecine, à la Faculté de médecine et de pharmacie de Lyon. À partir du deuxième cycle de formation médicale, nous avions la possibilité de nous initier à la recherche en y consacrant une partie de notre temps personnel. J'étais impressionné par l'enseignement que j'avais reçu en physiologie auprès de mon maitre Joseph Chantonnet, auprès de son propre maître le professeur Hermann, et auprès de JeanFrançois Cier et d'autres, mais c'est surtout auprès de Joseph Chantonnet. C'est intéressant parce que j'ai dû découvrir comment la formation passe par 
une sorte de rite initiatique. Il y a une première étape où, quand on est reçu, on doit s'impliquer dans certaines tâches pour participer à un programme de recherche et s'initier à une tâche de recherche. On essaie de s'introduire et de rendre service de façon à être intégré. Il s'agit donc de s'impliquer et de travailler en acceptant parfois les tâches ingrates dont personne ne veut. Au cours de cette étape, j'étais avec des chefs d'équipe extraordinaires comme mes amis Michel Cabanac et Yves Minaire, qui secondaient le professeur Chantonnet. Dans son laboratoire, il n'y avait pas de problèmes de différences culturelles. Pour la formation, il y avait le professeur So Sata, originaire du Cambodge, qui est maintenant revenu à Lyon. Il aimait bien la diversité culturelle. Pour lui, c'était un enrichissement, même dans une équipe de recherche en biologie, en physiologie. Et la physiologie, c'est important parce qu'on étudie les mécanismes de régulation biologique, le fonctionnement normal de l'organisme. C'est la meilleure façon de comprendre la pathologie si on comprend les processus normaux et les processus de régulation. C'est très important. D'ailleurs, avec Bateson plus tard, la théorie de la régulation sera même introduite en sciences humaines. Bon, il y a eu des excès avec ces théories et leurs répercussions sur les sciences humaines, mais quand même c'était important qu'elles soient introduites. En tout cas pour moi, dans mon expérience personnelle, c'était important que je commence par la physiologie.

Au cours de cette initiation, la seconde étape est lorsqu'on est retenu, du fait du travail qu'on a fourni, pour figurer dans une publication. Ça signifie qu'on devient un collaborateur, qu'on est reconnu comme associé d'un programme de recherche. C'est très intéressant parce qu'en plus, dans une recherche biologique, nous ne sommes pas dans l'imaginaire idéologique. En fait, ou on réussit ou on ne réussit pas l'examen de passage d'une étape à une autre.

Après, il y a une autre étape, c'est celle où on devient responsable d'une recherche sous la direction de quelqu'un. C'est toute la responsabilité de la thèse. Il se trouve - c'est un hasard - que j'ai eu à réaliser mon projet et discuter fréquemment de son avancement avec le patron lui-même, le professeur Chantonnet. Mon travail portait sur les problèmes d'adaptation au stress thermique. La thermorégression est la fonction biologique qui a été acquise la plus tardivement. De ce fait, dans des situations extrêmes de stress thermique, elle met à son service toutes les autres fonctions (cardiovasculaire, respiratoire, etc.). C'était pour moi une surprise. Beaucoup de chercheurs - entre autres des Canadiens - travaillaient à l'époque sur le stress thermique. Il y avait également, à New Haven, le professeur Hardy. C'était un grand 
monsieur. J'ai eu le plaisir de le connaître alors qu'il était le chef du département qui dirigeait tous les projets de recherche sur la thermorégulation à New Haven, aux États-Unis. Nous avions des contacts avec plusieurs universités nord-américaines, notamment avec le professeur Louis Leblanc qui est à l'Université Laval, le professeur Proulx, le professeur Florent Despocas, J. Sanford Hart qui était le chef d'équipe à l'Université de Toronto. Il faut dire que ce type de recherche intéresse beaucoup, y compris les militaires parce qu'elle touche aux problèmes de survie dans des conditions de stress thermique. Les Allemands avaient d'ailleurs déjà commencé cette recherche dans les années 40-45.

Lorsque nous avons commencé, c'était une recherche de pointe et notre laboratoire était un laboratoire de référence. Ce qui était extraordinaire, c'était de faire avancer la recherche dans ce domaine en redécouvrant que la biologie offre déjà un modèle, certes moins complexe que le modèle psychique, mais tout de même un modèle simplifié de la régression adaptative, de la régression non pas en tant que trouble de l'évolution, mais en tant que mécanisme positif d'adaptation au stress. La notion de stress n'est pas seulement à entendre dans le sens négatif d'un trauma ou d'un trouble du développement; elle peut au contraire témoigner d'une aptitude au développement puisque, devant un stress important qui met en jeu les conditions de survie, il est nécessaire d'avoir les possibilités de régresser à des mécanismes adaptatifs, même très anciens dans la phylogenèse.

Par exemple, l'animal exposé à un froid très intense - par rapport à ses possibilités de produire de la chaleur pour se réchauffer et par rapport à ses capacités d'isolement thermique - se met à frissonner pour produire la chaleur. À ce moment, il va vaquer à ses occupations, marcher, chercher sa nourriture et donc, se mobiliser pour produire de la chaleur avec ses muscles. Quand il y a compétition entre deux besoins fondamentaux, celui de se nourrir et celui de se réchauffer en maintenant sa température interne à un niveau donné, il faut trouver des moyens. C'est à ce moment que l'animal régresse à une modalité de production de la chaleur qui ne nécessite pas d'activité musculaire, qui se développe dans le muscle, qui se développe dans le tissu adipeux. C'est un tissu adipeux qui n'est pas comme un tissu graisseux habituel, qui n'existe que chez les nouveaux-nés. Même chez le nouveau-né humain, il existe un peu. Et ce tissu graisseux sert un peu comme s'il remplaçait le système hormonal archaïque: il est à la fois source de chaleur et moyen pour stimuler une production de chaleur qui ne nécessite pas la mobilisation du muscle. De la même façon, il y a une adaptation des moyens d'isolement 
thermique. On voit par exemple que des animaux comme le rat et le hamster, lorsqu'ils sont soumis de façon intermittente ou permanente à un froid aigu, sont vraiment adaptés au bout de quatre semaines. Mais au bout de quatre semaines, plus le froid et les conditions de froid auxquels ils sont exposés sont intenses, plus ils régressent à des modalités archaïques, néo-natales de production de chaleur.

Donc, quand on dit que quelqu'un fonctionne sur un mode archaïque ou sur un mode régressif, cela ne signifie pas qu'il soit lui-même archaïque. Cela signifie tout simplement qu'il vit dans des conditions stressantes qui font que, heureusement pour lui, il régresse. Autrement, il ne pourrait pas survivre. Et je crois que cette notion est importante, parce qu'il est très utile de pouvoir utiliser cet éclairage biologique pour mieux aborder ce qui est déjà dit sur le caractère adaptatif de la régression, mais d'une façon floue, en matière de régression psychique par exemple. Voilà.

J'étais donc à mon troisième stade initiatique puis je suis passé au quatrième. Je pouvais enfin avoir un programme de recherche et je pouvais en choisir le sujet. J'avais aussi un budget pris à même les subventions attribuées par le département à notre laboratoire. À cette époque, j'étais déjà en formation en psychiatrie. J'étais parvenu à un stade avancé et je vivais une situation intéressante qui m'a beaucoup aidé car au moment où je commençais à avancer dans cette formation, j'étais déjà à un stade où j'avais atteint la quatrième étape initiatique si vous voulez, celle d'avoir mon propre sujet de recherche. En fait, mon sujet de recherche était un peu excessif parce qu'il s'agissait de quatre enfants psychotiques... C'était dans le service de mon maitre Docteur Beaujard qui était un homme très cultivé mais très modeste, d'une très grande valeur. C'est lui qui s'occupait de la pédopsychiatrie à l'hôpital de Lyon.

Pour cette recherche, j'ai donc étudié le cas de quatre enfants, successivement... Deux dans un premier temps et ensuite, en faisant bien sûr attention à ce que m'avaient révélé les deux premiers, nous avions découvert deux autres cas similaires sur une période de six mois... À la suite de la suspension des visites de sa maman - elle avait ses raisons - dans l'institution où il était né, l'un des enfants avait ressenti cela comme un abandon. Même s'il était attaché à une éducatrice et à une infirmière, ces soignants ne remplaçaient pas sa maman pour lui. Ça, c'est intéressant. Il fallait que sa maman, qui venait régulièrement le voir, ne le voie plus pour qu'il régresse, mais d'une façon terrible. Charles Bingham et Anna Freud avaient décrit des situations homologues où des enfants, qui avaient un vécu d'abandon brutal par rupture ou par suspension de visite familiale, régressaient de cette façon et devenaient 
agités et agressifs, ou se mettaient à se balancer en suçant leur pouce et en faisant des onomatopées un peu stéréotypées, en ronronnant un peu d'une façon également très stéréotypée. Ils se salissaient aussi, il fallait leur mettre des couches, et cetera, et cetera. Ils régressaient vraiment aux stades premiers, comme s'ils venaient de naître, comme s'ils étaient des bébés de quelques mois, de quelques semaines ou même de quelques jours. Donc, la maman de l'un des enfants ne venait plus et l'éducateur ou l'éducatrice qui jouait le rôle de substitut maternel, d'image maternelle, était épuisé(e) et avait pris congé sans que l'institution y soit préparée. Dans de tels moments, l'enfant vit un abandon brutal et une régression. Que peut-il alors se passer?

Un de ces enfants s'est mis à côté du chauffage. En fait, ils se mettaient tous à côté du chauffage en chien de fusil ou bien se couvraient avec une couverture. Il arrive souvent dans les hôpitaux psychiatriques que le chauffage soit en panne ou soit trop chaud jusqu'à être brûlant. Et l'un des enfants s'est brûlé une jambe et un genou. Or, même après le traitement de ses brûlures, il continuait! Et c'est ça qui était déroutant: il continuait à se mettre à côté du chauffage d'une façon symbiotique, d'une façon un peu fusionnelle. Un autre avait un chat, mais tu sais comment sont les chats des psychotiques: ils se laissent manipuler, ils deviennent vraiment des chats objet, ou bien c'est la couverture... Mais ce n'est pas l'objet transitionnel de Winnicott; dans ce cas, c'est une couverture qui sert d'enveloppe en remplacement de la maman. En travaillant sur la thermorégression, j'avais formulé une hypothèse et je me suis dit qu'on pouvait la vérifier.

Il y avait à l'époque le travail de Jacques Cosnier, qui reprenait le travail de Harlow à Lyon. Ce dernier avait établi le fait que plusieurs animaux/nouveaux-nés, quand ils étaient élevés avec des mères artificielles, préféraient le contact de la mère/fourrure plutôt que celui de la mère/métallique même si la source de nourriture était la mère/métallique. Aux États-Unis, Rosenblatt et Snellen travaillaient sur les premiers comportements du nouveau-né, notamment sur le comportement alimentaire et la succion réflexive en utilisant eux aussi des mères artificielles. L'idée était la suivante: s'il manque à ces enfants la couverture en laine à laquelle ils se colleraient symbiotiquement et s'ils se collent sur le radiateur même s'il leur fait mal et les brûle, cela signifie qu'ils régressent à quelque chose d'antérieur à la capacité de sentir la douleur physique puisqu'ils ont une douleur encore plus profonde qui est celle de l'abandon, du désarroi, de ce qui a été appelé le vrai traumatisme de la naissance. Les enfants régressent donc au niveau de l'effondrement comme dit Winnicott, d'une agonie. Pour échapper à cette agonie, je me suis dit qu'ils 
doivent chercher à rétablir leur homéothermie et je me suis dit qu'ils doivent avoir régressé à un stade néo-natal, à un stade où on sait que, à la naissance, tous les homéothermes, et davantage l'humain, sont immatures.

Le nouveau-né humain naît à un stade encore plus immature que plusieurs animaux mammifères et, à la naissance, il fait face à un double problème. D'abord, il ne possède pas beaucoup de capacité à produire de la chaleur et deuxièmement, sa masse est trop faible par rapport à l'étendue de sa surface de distribution de chaleur. Ses moyens d'isolement thermique sont très déficients et sa capacité de production de chaleur, très faible. Par rapport à une température interne moyenne de 37 degrés qu'il doit maintenir stable, une température de 28 degrés peut être froide. Il lui faut beaucoup d'énergie et de chaleur. Les enfants psychotiques dont je parlais régressent à ce stade néo-natal où ils recherchent dans la mère/fourrure ou la mère réchauffée, qui apporte la chaleur, une enveloppe compensant les déficits en isolement thermique et qui, surtout, apporte la possibilité de ne pas perdre sa chaleur et de se réchauffer.

Alors, c'était simple. Suite à ce qu'avaient démontré ces quatre patients, nous avons bâti un protocole expérimental dans le laboratoire en utilisant plusieurs modèles de nouveaux-nés animaux. Nous avions effectivement constaté que si nous réchauffions la mère/métallique et qu'au contraire, nous refroidissions la mère/fourrure, nous avions alors une inversion du phénomène qui avait été découvert par Harlow. Si la mère/fourrure est refroidie par le passage d'un liquide réfrigéré en dessous de la fourrure - 20-25 degrés, ce qui est froid pour un nouveau-né - et qu'on réchauffe la mère/métallique, le nourrisson de toutes ces espèces préfère le contact du métal qui apporte un réchauffement plutôt que le contact de la fourrure. Par conséquent, ce n'est pas une question de contact en lui-même avec la fourrure, c'est une question de recherche d'une mère/enveloppe qui compense son déficit, la mère étant pour le nouveau-né son enveloppe thermique.

Autrement dit - et cela est tout à fait en accord avec Spitz et tous ceux qui ont travaillé sur l'enfant -, la mère n'existe pas en tant que telle; elle ne va être différenciée en tant que maman et personnalité, même chez le nouveau-né humain, que quelques semaines après la naissance. Dans un premier temps, la mère est simplement une enveloppe thermique. Mais le bébé n'est pas inactif comme on le pensait. Il est très actif et cherche cette maman pour s'y attacher de manière fusionnelle en tant qu'enveloppe, en tant que partie de lui-même. Nous avons là un éclairage sur cette zone pleine d'ombre et d'obscurité dont parlait Freud quand il disait que tout commence par l'oralité dans 
le développement psychique. Il a quand même laissé la question ouverte. C'est ça son génie théorique. Il a dit «oui, mais attention! Il y a quand même peutêtre quelque chose avant l'oralité, il y a une zone pleine d'ombre et d'obscurité. Qu'est-ce qui se passe pour articuler le biologique au psychique?» MP : Plus archaïque que l'oralité.

EJ : Plus archaïque. Ces enfants psychotiques - effectivement régressés du fait de l'abandon brutal par leur maman ou leur substitut maternel - vivaient une angoisse d'effondrement. Pour survivre à cette angoisse, ils régressaient à un stade antérieur, au stade néo-natal par lequel ils mobilisaient une visée pulsionnelle-biologique allant vers la mère/enveloppe, vers la mère/archaïque, cette mère non personnalisée qui est une simple enveloppe thermique. C'est à partir du moment où l'enfant va vers cette mère archaïque qu'il manifeste une réceptivité active, et non pas passive, pour ce qui va s'engrammer en lui à travers la maman en tant qu'enveloppe. Celle-ci devient alors, comme dit Joyce McDougall, le support de l'inconscient du bébé. L'inconscient du nourrisson, avance-t-elle plusieurs fois, c'est la maman qui le porte et c'est la maman qui le transmet. Et comme le dit cette fois Piera Aulagnier, il y a engrammation d'éléments hétérogènes; à travers une pulsion unitaire, on voit apparaître le jeu. Mais cette pulsion unitaire, au départ biologique, c'est la pulsion homéostasique-homéothermique. C'est elle qui cherche à regrouper les fragments inscrits et qui entraîne l'émergence d'un jeu à partir duquel le nourrisson va exercer son mécanisme de fonctionnement paranoïde, comme dirait Mélanie Klein. Pour ma part, je préfère parler avec Winnicott de sa capacité à être seul, à actualiser son être omnipotent dans un cadre impliquant la capacité d'être seul.

MP: Qu'est-ce que vous appelez, vous, un être omnipotent?

EJ : À ce stade, c'est au niveau du "Je suis», au niveau de l'être que le Je émerge, que le Je agissant, que le Je en tant qu'agir va s'exprimer, s'extérioriser en tant qu'être. C'est le «Je suis» qui précède le «Je pense». Ce n'est pas le «Je pense donc je suis», mais c'est le «Je suis donc Je pense». J'ai commencé à penser cela alors que j'étais à un stade initiatique: je commençais mon analyse alors que j'étais plongé dans la physiologie, la psychophysiologie, la neurophysiologie. J'étais déjà à un stade très avancé de ce côté puisque j'étais non seulement maître de ma recherche, mais aussi maître de mon programme de recherche.

MP : C'est à ce moment, déjà, que vous avez commencé à articuler d'une façon relativement dynamique, pour ne pas dire très dynamique, le roc du biologique, comme disait Freud, au déploiement du psychisme à l'intérieur 
d'une réflexion sur la régression et la régression positive. Tout ce qui est du domaine de l'archaïque n'est pas versé du côté de la négativité, mais plutôt du côté de la constitution du sujet, de l'être dont vous parliez, et en même temps de la constitution progressive à travers l'unité hétérogène. Il y a donc là quelque chose qui permet le déploiement progressif de la psyché.

EJ : Tout à fait. C'est cette pulsion invisible homéothermique, au départ purement biologique...

MP : Si vous me permettez, je vous interromps pour dire que je crois que je c'est ça qui est particulièrement original, cette pulsion à visée homéothermique. Il y a là une condensation extraordinaire.

EJ : C'est une pulsion biologique. Mais vous savez que même chez Freud, et même après Freud, le concept de pulsion a toujours été ambigu.

MP: Oui, c'est un concept limite!

EJ : C'est un concept limite qui a toujours été ambigu. Je dirais qu'il y a un niveau où la pulsion est biologique, mais qu'elle ne va pas rester longtemps biologique. Trois jours, quatre jours, cinq jours parce que les choses changent chez un nourrisson très rapidement. Donc, c'est une pulsion à visée biologique, mais quand la maman va répondre... il y aura déjà une différence. Dans mes travaux, je distingue entre une maman vivante et une maman artificielle. Ce n'est pas le même type de réponse.

MP: Qu'est-ce que vous appelez une maman artificielle justement?

EJ : J'ai parlé de la maman artificielle dans mes premières publications sur le sujet en 68 puis le travail complet a été rédigé en 69. Comme il faut une année entre l'acceptation d'un travail dans une revue internationale et sa publication, il a été publié en 70. Je l'avais envoyé à Bowlby en 69. Je l'avais aussi envoyé à Rosenblatt et à Snellen aux États-Unis, qui m'avaient envoyé d'autres travaux. Bowlby ne m'avait pas répondu, mais c'est vrai que je ne sais pas s'il l'a repris totalement ou en partie. Lorsqu'il parle de l'attachement, il parle de la chaleur de façon un peu floue et je pense que ce concept aurait pu avoir plus de poids s'il avait intégré le facteur thermotactile en tant que mécanisme premier de l'attachement, de la constitution de cet attachement.

MP: Oui, d'autant plus que chez Bowlby, la pulsion disparaît d'une certaine façon en faveur de quelque chose qui est d'ordre purement intersubjectif. La pulsion thermorégulatrice dont vous parlez et qui forme le socle, le creuset devrais-je dire, sur lequel vont s'organiser les modalités pulsionnelles du nouveau-né, en vient finalement à être éliminée.

EJ : Oui, parce que le nouveau-né humain n'a, dès la naissance - et cela est également vrai pour le nouveau-né animal -, qu'une seule vraie action 
orientée: c'est cette réaction visant une source de réchauffement ou une enveloppe qui à la fois apporte un réchauffement et un complément d'isolement. MP: C'est le versant actif.

EJ : C'est la seule action qui soit orientée. Les autres besoins sont là comme stimuli et réponses à d'autres stimuli nociceptifs, comme à des besoins internes non orientés. Il s'agit de facteurs hétéroclites, même pulsionnels, mais hétéroclites. Donc, seule compte la visée pulsionnelle biologique pour établir ce lien avec la maman. Bien sûr, la maman vivante, humaine ou animale - comme le montrent certains travaux de 70, de 72 ou 77 -, est conditionnée à accepter de rester immobile, de ne pas trop bouger quand son nouveau-né commence à chercher le mamelon. Elle est là, vivante, mais il y a un côté en contention de façon à pouvoir faciliter un peu le travail. C'est vrai qu'il y a certaines réserves, mais déjà, même sous contention, on a pu démontrer qu'elle répond activement. Elle ne va pas simplement jouer son rôle d'enveloppe protectrice, elle va également satisfaire le besoin de nourriture et cela, d'autant plus qu'il y a un double sens. S'il n'est pas nourri, le nouveau-né tombe en hypothermie alors il s'oriente normalement vers la région la plus chaude de l'abdomen de la mère lapine ou de la mère chienne, ou de tous les modèles animaux qu'on a étudiés. Quand on fait des thermographies, la région la plus chaude chez une mère allaitante est la zone du sein, où il y a la montée lactée. La petite bête n'est pas bête: tout en étant un nouveau-né à un stade très archaïque, il s'oriente progressivement en cherchant, grâce à son museau et ses thermocepteurs, la zone la plus chaude qui se trouve être la zone mammaire. Et là, la maman est active pour l'entraîner vers le mamelon et l'aider un petit peu à trouver sa place puis à téter et à s'alimenter. Elle répond non seulement à son besoin homéothermique, mais aussi à son besoin de nourriture.

C'est très typique chez les singes nouveaux-nés: chaque fois que quelqu'un s'approche et que la maman singe vit cela comme un danger pour son enfant, elle réagit d'une façon très agressive pour le défendre, pour être son enveloppe protectrice, vis-à-vis tous les stimuli. Elle est à la fois celle qui apporte réponse à tous les stimuli et qui protège de tous les stimuli nociceptifs. C'est pourquoi tout ce qui est protection se condense sur le facteur thermotactile, ce qui explique que lorsque quelqu'un se sent en situation d'abandon ou d'insécurité, il décrit cette situation en termes de froid.

MP : Fedida parlait d'ailleurs de la dépression comme d'un affect glaciaire. EJ : Voilà. C'est à ce niveau que s'articule le processus originaire. MP: Comment distinguez-vous votre position de celle de Didier Anzieu? 
EJ : Je n'ai pas de réponse pour Anzieu. Vraiment! Je ne l'ai même pas lu. Je l'ai jeté parce que je suis sûr de sa mauvaise foi.

MP : D'accord, même s'il serait intéressant de voir l'articulation entre la théorie du moi-peau et de sa célèbre mère. Allons donc plutôt du côté des processus originaires.

EJ : Alors là, ça va se différencier progressivement: le facteur thermotactile va devenir un facteur oral. C'est sur une pulsion biologique que va s'articuler à travers la maman, à travers ce que la maman apporte comme engrammation, le culturel...

MP: Alors, finalement, dans vos recherches, vous mettez en lumière et corroborez l'intuition freudienne selon laquelle le déploiement du psychisme humain, très très tôt dans l'histoire du sujet, s'étaye sur le roc du biologique, ce que vous appelez la pulsion thermotactile. Est-ce que j'ai bien compris? EJ: Oui. La pulsion thermotactile, au départ, est biologique sauf que, à partir du moment où je l'appelle facteur thermotactile, il n'est plus biologique. MP : Est-il déjà psychique?

EJ : Oui, il est déjà psychique. Il est déjà inscrit dans le culturel puisque la maman, en tant qu'enveloppe, a déjà commencé à répondre à la demande de son enfant, une demande dénuée de figurabilité. C'est une demande archaïque. La mère ne répond pas seulement aux besoins de réchauffement et d'isolement thermique, elle répond en protégeant le nouveau-né de tous les stimuli nociceptifs, en facilitant son alimentation... en le nettoyant de façon à ce qu'il n'ait pas froid aussi parce que s'il n'est pas nettoyé, il perd sa chaleur. À partir de là, ce que la maman a engrammé en lui, même s'il est dénué de figurabilité, comme le dit très bien Piera Aulagnier, est déjà au fondement de ce que Paul Federn appelle l'articulation première. Je suis tout à fait d'accord avec lui quand il dit qu'on doit se rendre compte que la pulsion affleurant dans le travail avec le psychotique met en évidence le fait que, très précocement chez l'enfant, il y a l'articulation du culturel. Il a fallu Freud, il a fallu Federn, il a fallu Piera Aulagnier, il a fallu Gisela Pankow, Joyce McDougall et Winnicott pour comprendre cela. Il y a une visée au départ, en tant qu'homéothermique, une pulsion biologique en tant que comportement thermorégulateur. Le seul comportement orienté qui puisse diriger l'enfant vers la maman, la réponse de la maman, c'est non seulement qu'elle soit une enveloppe protectrice réchauffant et isolant des risques d'hypothermie, mais qu'elle réponde en satisfaisant d'autres besoins qui vont automatiquement s'articuler avec le besoin de réchauffement, qui vont se condenser avec ses besoins et condenser autour d'elle tous les besoins de protection. C'est 
justement ce qui explique que, comme je le disais tout à l'heure, au moindre stress ou à la moindre sensation d'effondrement dans les événements de la vie, tout un chacun peut découvrir la réémergence en lui, face à un danger ou un effondrement imminent, de cette sensation de froid ou d'un accueil froid, d'un environnement froid ou une sensation de frayeur liée au froid. C'est le concept d'effroi. Lorsque les habilités se détachent des facteurs thermotactiles pour devenir indépendantes, à partir du moment où l'enfant découvre qu'il peut agir sur sa maman en acceptant ou en refusant la nourriture - ce qui correspond classiquement au stade oral actif -, en jouant ou en ne jouant pas avec le mamelon, nous sommes dans le culturel. Ce que je voulais dire, c'est qu'avec l'oralité, nous ne sommes plus dans le modèle de la protection par rapport à l'angoisse d'effondrement, nous sommes dans le modèle des rapports d'échange enfant-maman et maman-enfant.

MP: Quelle place faites-vous dans cette perspective au sexuel au sens freudien du terme, à l'aspect libidinal et donc, si je prolonge ma question, quelle est la place dans ce processus que vous décrivez du fantasme? Je pense ici particulièrement au fantasme maternel vis-à-vis de l'enfant.

EJ : Je vous répondrais en attirant l'attention sur la clinique des troubles du post-partum. Je parle des troubles de post-partum purs, de nature psychotique, mais sans que cela soit une psychose. Six mois après l'accouchement et quelques mois après la fin de l'allaitement, il peut y avoir des rechutes sous forme de différents troubles, mais sans qu'il y ait de composantes psychotiques. Voilà la différence selon moi entre une psychose déclenchée par l'accouchement et les troubles purs du post-partum, même de nature psychotique. Ces derniers ne sont pas des psychoses déclenchées par l'accouchement, mais sont déclenchés par la grossesse, par la fin de la grossesse ou par l'accouchement, l'allaitement ou la fin de l'allaitement. Si après ces étapes, il n'y a plus de trouble, cela signifie que c'était une psychose post-partum.

Alors, si on étudie un petit peu, si on examine et on a un suivi thérapeutique pour des troubles du post-partum, on s'aperçoit de quoi ? On s'aperçoit que la maman a un rapport dyadique avec l'enfant. Ce qui pose problème, c'est que la grossesse ou l'accouchement de la jeune maman réveille en elle ses propres fantasmes par rapport à sa mère. Lorsque le rapport est ambigu, s'il y a beaucoup d'ambivalence dans ce rapport mère-fille, c'est là qu'il y a le plus souvent des troubles du post-partum. Et ces troubles montrent que, pour que le rapport mère-enfant soit un rapport de la maman avec son enfant, la thérapeutique doit conduire à ce que la maman commence à s'occuper de son enfant et à imposer à sa propre mère le fait que cet enfant est le sien et 
qu'elle a le droit d'être mère à son tour, pour elle-même et non pas pour sa mère. Tout le travail thérapeutique passe par là. On s'aperçoit que la maman répète les difficultés, normales à un stade donné, qu'elle a eues avec sa mère. Le rapport dyadique avec l'enfant fait que si l'enfant dans son système voit sa maman en tant qu'enveloppe, en tant que partie contenante de lui-même, la maman voit en son enfant une partie d'elle-même. Ce n'est pas une partie qui englobe une totalité, mais c'est une partie quand même... Et ce rapport dyadique facilite sa disponibilité à engrammer et à répondre presque fusionnellement à l'enfant. Ce n'est pas la même chose que la fusion, mais disons qu'il y a quelque chose de l'ordre de la recevabilité de la fusion de l'enfant parce que dans ce premier état, elle considère celui-ci comme une partie d'ellemême.

Même si cela est positif, il est important que ce rapport enfant-mère, mère-enfant soit très tôt contrôlé par un organisateur culturel. Très tôt aussi, à travers le langage de la maman qui parle, celle-ci devient la représentante de son enfant. Nous avons là le processus originaire et les perturbations potentielles de l'inscription de ce processus en fonction des difficultés maternelles. La mère inscrit ce processus originaire et dès lors, convoque l'organisateur culturel. Si la phase de rapport symbiotique maman-enfant et enfant-maman n'est pas régulée par l'organisateur culturel, on se retrouve avec des difficultés de processus primaires de personnation. Ceux-ci passent donc par l'articulation du biologique au culturel, mais ce biologique est tout de suite engrammé par le langage maternel, conscient et inconscient. Il est également tout de suite engrammé par le roman familial que porte la maman et toute la représentation familiale de la maman de cet enfant. S'il y a discordance entre le papa et la maman dans le projet de l'enfant, discordance dans la nomination de l'enfant, il y a déjà un problème inscrit dans le processus originaire de l'enfant.

MP: Vous touchez là à quelque chose qui me questionne depuis un certain temps, à savoir l'articulation de la dyade mère-enfant et de l'organisateur culturel. Je me demandais quelle est la place, dans ce processus, à l'intérieur de la culture et à l'intérieur de ce processus, du déploiement du psychisme, quel est la place du Nom-du-Père, pour parler comme Lacan? Où est-ce que vient s'inscrire cette fonction symbolique, cette fonction symboligène extrêmement importante pour que la fusion se défasse, mais d'une façon qui soit porteuse pour l'évolution de l'enfant?

EJ : Elle est dans deux choses. Elle est d'abord dans le discours de la maman, lequel indique que la maman se réfère elle-même à une image paternelle et 
à une figure paternelle qui représente pour elle l'ordre symbolique, qu'elle a inscrite dans l'ordre symbolique au niveau des processus d'historicisation. MP: Donc, dans les générations, dans la transmission.

EJ : Dans les générations au niveau des processus d'historicisation. C'est là où il $\mathrm{y}$ a une interrogation par rapport au discours féministe comme au discours anti-féministe. Je pense qu'il y a problème à chaque fois qu'il y a ce rapport de dualité alors que les choses sont plus complexes. De toute façon, toute maman ne peut être une maman telle que Winnicott l'a définie. Les définitions les plus simples et les plus sensées, les plus extraordinaires et les plus merveilleuses, sont celles données par Winnicott. Une maman ne peut pas être une mère biologique. C'est une maman qui, effectivement, à partir d'un socle biologique par lequel elle engendre un enfant, s'appuie elle-même sur un socle culturel, sur un roman familial, sur une façon d'avoir été historicisée elle-même et d'être historicisante pour ce qui va advenir de ses propres enfants quand elle en a. La problématique est donc: que fait-elle avec sa référence paternelle? Est-ce que c'est une référence à une figure figée de père ou est-ce que c'est une référence à un père symbolique? Si la figure se confond avec le symbolique, si la pensée est une pensée par images sensibles et de type iconologique, il y a problème, parce que la mère ne peut pas s'ouvrir à une autre figure paternelle que la sienne. Et c'est alors que l'organisateur culturel va fonctionner ou non.

MP: Donc, ce que vous dites finalement, de la même façon que Winnicott pouvait dire « un bébé, ça n'existe pas seul », c'est qu'une mère ça n'existe pas seule non plus.

EJ : Ça n'existe pas seule non plus. Et c'est extraordinaire qu'un peintre comme de La Tour, dans ce tableau merveilleux sur la maternité, nous montre la jeune maman portant son bébé langé et la grand-mère qui lui tient un peu l'épaule et la couvre, l'enveloppe pour qu'elle puisse elle-même envelopper et découvrir comment envelopper son enfant. Alors, la maman est comme l'enveloppe réchauffante, mais en même temps, elle tient une lumière qui participe à cette enveloppe réchauffante, à cette illumination de la vie et au rapport de la chaleur avec la lumière de la vie. Cette double dyade grandmère/mère/enfant est absolument importante et fantastique à travers toutes les cultures, c'est quelque chose de très structurant pour aider la maman à être une excellente mère - sachant qu'il n'y a pas de maman parfaite - qui sache simplement anticiper les besoins de l'enfant sans casser sa créativité... en étant attentive et en étant un environnement enrichissant pour sa créativité. 
MP: Donc, good enough équivaut à anticipatrice.

EJ : Tout à fait. Anticiper, mais ne pas anticiper d'une façon rigide, anticiper en laissant l'autre aller vers ce qu'elle avait anticipé. Anticiper dans l'ouverture, mais en laissant l'autre construire sa place et en étant un support pour son playing, son jeu, son jeu sans règle. Son playing et non sa game. C'est extraordinaire qu'il y ait en anglais ce double concept. Le nouveau-né investit l'espace, il joue et, en fait, il se découvre lui-même à travers son mouvement, son corps agissant. C'est cet agir qui lui permet de construire une unité entre les diverses parties qui le constituent. Et la maman doit lui donner la possibilité de construire ses fonctions symbolisantes, le lien entre la partie et le tout, à travers ce playing et à travers cet espace potentiel qu'elle lui fournit. Je suis tout à fait d'accord avec Piera Aulganier quand elle a dit avant tout le monde que les représentations de l'originaire, comme le fantasme de l'originaire, ne sont jamais de l'ordre de la pulsion, qu'ils sont secondaires à la pulsion, même s'ils sont dénués de figurabilité. Les fantasmes ne s'inscrivent pas sur le biologique. Le fantasme et les représentations fantasmatiques, tout ce qui est représentation, même quand ce sont des représentations archaïques, s'inscrivent et s'articulent au culturel à travers le langage de la maman et à travers ce que la maman, d'une façon consciente et inconsciente, a engrammé en son enfant en tant que représentant d'un projet familial dans le continuum familial ou en discordance par rapport à ce continuum.

MP: Vous faites souvent référence, par exemple dans Filiation et altérité, à la distinction entre l'originaire et l'origine. Vous parlez de fantasmes originaires, de processus originaires, mais en même temps, on a parfois l'impression que vous créez une sorte de passage ou en tout cas une articulation complexe certes, mais qui demande à être éclairée, entre ces deux dimensions. Il me semble comprendre que pour vous, l'origine serait davantage de l'ordre de l'imaginaire alors que l'originaire serait plutôt quelque chose qui est engrammé et fonde l'articulation du symbolique. Je ne sais pas si j'entends correctement, mais l'origine ferait référence à la dimension mythologique, n'est-ce pas, alors que l'originaire, comme articulation au symbolique, intègrerait la dimension espace-temps. Bref, est-ce que vous pourriez éclairer un peu ces deux notions pour nous? Parce que c'est ce qui nous permet finalement de comprendre, et nous y viendrons ensuite, votre travail avec les psychotiques.

EJ : Bon... moi je n'en parle pas d'une façon théorique, philosophique... mais j'en parle à partir d'une démarche pratique. Ce qui nous interroge à partir de la prise en charge au long cours d'un patient psychotique ou d'un groupe de patients psychotiques, ce que cela implique comme moments difficiles pour 
le thérapeute quel qu'il soit, ce que cela implique de remises en question, d'hésitations, d'interrogations sur certains concepts, d'interrogations sur notre ignorance à partir desquelles nous sommes sollicités afin d'approfondir notre réflexion, notre savoir et nos échanges avec les uns et les autres de nos collègues... C'est que nous avons besoin, d'une façon opératoire, à la fois du concept et de l'usage du concept d'origine, mais nous avons encore davantage besoin, comme thérapeute, des concepts de processus originaire et de refoulement originaire. Ceux-ci sont un passage obligé même si on peut plus ou moins négliger l'origine... surtout que les idéologies ne parlent pas de processus originaires, elles ne parlent que des origines et inscrivent l'histoire des gens dans une origine. C'est comme si l'espace pouvait contenir le temps, c'est une inversion des données. Plutôt que le temps soit le contenant, dans le management moderne et le comportementalisme de l'espace, on met des morceaux, des fragments d'histoire. Déjà ce management c'est un petit peu "psychotigène». Je crois que nous ne pouvons pas nier la question de l'origine à cause de cela, de cette approche critique, mais disons que l'origine, ça reste au niveau où l'idéologique fonctionne. C'est parce qu'elle utilise la mythologie et la pensée iconologique et confond les symboles et le symbolisme comme figurabilité, la pensée par images sensibles et la pensée iconologique, qu'il y a confusion.

De mon point de vue, l'origine fait essentiellement référence à l'espace en tant que contenant. Pour faire référence à l'espace en tant que contenant et introduire le temps, nous avons besoin de construire un mythe. Tout roman énonciateur, tout discours énonciateur peut se passer au niveau du discours mythologique, au niveau du discours et du récit historique. Je pense donc qu'une distinction entre les deux est nécessaire. L'originaire est un processus inscrit dans le sujet qui, du fait de cette inscription, est porteur d'une histoire dans son côté historicisé et dans son côté historicisant; il inscrit l'enfant en tant que projet avant même qu'il soit conçu, il est un projet pour les parents. On pense à son avenir, on pense à le nommer, on pense à des représentations multiples, certaines sont conscientes, d'autres inconscientes. Son arrivée remet à jour un récit familial sur les possibilités d'accessibilité qu'on peut offrir à cet enfant au niveau du registre symbolique de l'avoir, du savoir, du pouvoir et de l'agir. Et sur le devenir qu'on a soi-même à travers lui dans le cadre d'un rapport de filiation assez complexe où l'enfant est enraciné en nous, mais nous, nous sommes aussi enracinés quelque part en cet enfant projet. Ce dernier est porteur d'une histoire très chargée dont une partie est investie sur le plan conscient, de l'ordre du mémorisable, alors qu'une autre 
partie est de l'ordre d'un récit refoulé. Cette partie est de l'ordre de ce que Piera Aulagnier appelle le mémorisé mémorisant, quelque chose qui est là dans notre histoire même, avec des éléments archaïques qui sont présents sans être là, qui restent actifs, des éléments de notre propre histoire, de l'histoire de chacun des parents, qui contribuent justement à enrichir et à donner une certaine épaisseur au récit, lequel est là sans être là. C’est ça qui ouvre aux possibilités de symbolisation, à la fois sur l'imaginaire fécond, mais aussi articulé au réel et à travers un symbolique, mais un symbolique riche et multiple... offrant une multiplicité. Le processus originaire, comme les perturbations de ce processus, et comme le refoulement qui peut aussi être là dans ce processus originaire, quelque chose de l'ordre du refoulé chez l'un ou l'autre des parents ou chez les deux parents, tout cela peut être porté par la maman qui va l'inscrire chez l'enfant. Tout cela, ce n'est pas l'origine et ça fait partie de l'histoire et de la construction de l'histoire... de l'histoire déjà faite, de l'histoire se faisant et se construisant. Tout individu a besoin, pour reconstruire son histoire, d'un mythe ou d'une part de mythologie. C'est pourquoi il y a cette nécessité du passage de l'historique au mythologique, donc à l'origine... à la frontière de laquelle, nous construisons notre récit historique. C'est là que le mythe envahit notre récit historique et que nous le confondons avec le récit historique. À ce moment-là, nous ne sommes pas dans une position thérapeutique. Au contraire, nous pouvons occuper la position de guérisseur ou, si nous sommes dans celle du scientifique, de thérapeute-scientifique qui fait référence à la science et à la psychanalyse en tant que science. Dans ce cas, nous sommes dans la déconstruction et la reconstruction du récit jusqu'à ce que le récit historique, historicisant et historicisé se construise et assume les difficultés de sa construction, mais se construise progressivement, se déconstruise, se reconstruise en s'appuyant sur une relecture toujours partielle de l'originaire... non pas de l'origine, mais de l'originaire... et une construction toujours partielle de la filiation symbolique qui articule et représente une sorte de lien entre les trois dimensions de l'identité: l'identité ressentie, l'identité reconnue et l'identité culturelle, qui prend place dans la dynamique entre les deux. Nous avons toujours besoin d'un espace d'origine à partir duquel nous nous projetons quelque part de façon mythologique, et à partir duquel nous construisons notre récit historique. En construisant celui-ci, nous réduisons l'espace du récit mythologique sans l'effacer complètement.

MP: Essedik Jeddi, tout ce que vous nous avez exposé jusqu'à présent démontre à quel point vous considérez - autant dans toutes les questions concernant 
le facteur thermotactile, l'étayage et les pulsions - l'importance du travail de culture pour le futur de nos civilisations.

EJ : Je pense qu'il est important. Nous disposons aujourd'hui de données biologiques de plus en plus importantes, pour parler de la dimension disease de la psychose par exemple et de toutes les psychoses et de toutes les dépressions. Mais ce qu'il y a, c'est que le malade est là et que notre pratique est là; la pratique des psychiatres, et pas seulement des psychanalystes. Bref, nous avons beau posséder des connaissances de plus en plus vastes au sujet de la dimension disease des maladies mentales et concernant la réponse des patients à tel ou tel type d'anti-psychotique ou anti-dépresseur, les résultats sont là pour démontrer que cela ne suffit pas. Aucun patient ne peut être traité de manière efficace uniquement avec des médicaments. Les familles, les patients, tout le monde est d'accord là-dessus. Nous assistons en outre aujourd'hui à des dérives en matière de prise en charge au long cours des malades psychotiques qui font que ces gens en état de souffrance sont abandonnés à la seule chimiothérapie ou à des thérapies qui n'impliquent pas un thérapeute, indépendamment des étayages théoriques. Des thérapies qui n'exigent pas que le thérapeute soit questionné et assume le questionnement que lui imposent les difficultés avec son malade et les difficultés qu'éprouve son malade au cours de la prise en charge. Tout cela fait que nous assistons à l'émergence d'un climat sécuritaire et d'une approche sécuritaire de la psychose. D'une part, nous constituons des équipes au sein desquelles les tâches sont compartimentées entre divers spécialistes et nous n'acceptons pas l'idée qu'il puisse y avoir un savoir en partage entre des spécialités aussi variées que celle de l'infirmière, celle de l'éducateur, celle du psychomotricien, ou celle du psychanalyste... MP: Ou de l'ergothérapeute...

EJ : Oui. Une équipe est multidisciplinaire. Mais une équipe multidisciplinaire, est-ce que cela signifie une équipe composée de disciplines compartimentées? Ou est-ce une équipe qui fonctionne sur un mode d'équipe, d'une vraie équipe multidisciplinaire avec un champ de savoir et un champ d'interrogation et de questionnements en commun, dont les membres acceptent qu'une part du savoir soit partagée, même si le savoir sera plus pointu dans telle catégorie de spécialités plutôt que dans telle autre. Car le malade psychotique est là pour nous interroger, pour nous demander: " mais où allezvous?» Pour nous dire, «mais écoutez, le médicament vous avez beau me le donner, il est bien, il coûte plus cher, mais cela ne suffit pas. » Et il nous dit à partir de quelle place il nous interpelle. Gisela Pankow a une phrase merveilleuse : elle dit que le patient psychotique, le schizophrène, le plus en 
retrait, apparemment il est en retrait, apparemment il ne dit rien, mais il attend, il fait mille appels... Je cite ça de mémoire... mais elle dit qu'il n'attend qu'une parole pour que son expression se mette en oeuvre, pour reprendre une expression de Husserl, pour que son expression se mette en oeuvre en tant qu'acte de signification ouvert sur une transcendance de sens, sur un horizon de sens. J'ai à l'époque proposé le concept de socioanalyse, mais actuellement, je crois que beaucoup de psychanalystes acceptent l'idée que la psychanalyse doive être un travail de culture. Dès lors que tout le monde accepte cela, nous n'avons pas besoin d'un concept nouveau.

Déjà, en 1932, Federn disait qu'il avait eu le mérite d'être le premier à s'occuper et à appliquer la démarche psychanalytique à la psychose, à la prise en charge au long cours des psychotiques. C'est lui qui avait eu l'idée géniale de dire que ce n'est pas au psychotique de s'adapter à la technique psychanalytique, mais que c'est à la technique psychanalytique, si c'est une théorie valable, de s'adapter. Il avait discuté de cela avec Freud, dont il était très proche. C'est à la psychanalyse de s'ouvrir et d'adapter sa méthodologie pour pouvoir prendre en charge le malade psychotique. À partir de cette ouverture de Federn, Freud s'est lui-même ouvert et lui recommandait des malades. Depuis, il y a quand même un certain nombre d'auteurs - je pense à Stern, Kohut et Gisela Pankow que j'ai connue et qui m'a apporté beaucoup, même lorsque j'ai eu à affronter le questionnement du psychotique tunisien, déjà en France, lorsqu'il m'a interpellé en disant: "Mais à partir de quelle place m'écoutes-tu?» Si je suis, du fait de ma culture «non occidentale», représentant des cultures ayant des pratiques de savoir pour lesquelles la science n'est pas accessible, la question est de déterminer si nous devons avoir des catégories scientifiques en fonction des espaces culturels, en fonction de cette mythologie de l'espace. Nous avons par exemple d'un côté la science comme knowledge et de l'autre côté le traditional knowledge. Alors, de quelle place est-ce que j'écoute mon patient? Suis-je là comme répétiteur ou agent programmé d'un savoir qui m'est extérieur? Si c'est le cas, le psychotique ne peut pas avancer avec moi. Non seulement je ne peux rien lui apporter, mais je pourrais être plus pathogène. Comment l'aider à reconstruire un discours historicisé et historisant, comment l'aider à reconstruire sa première fonction symbolisante, à faire le lien entre la partie et le tout, et sa deuxième fonction symbolisante, à pouvoir faire le rapport entre les différentes parties qui le constituent pour faire une totalité? Comment donner un contenu et un sens à ce lien afin qu'il puisse habiter son corps, afin qu'il puisse être sujet de son histoire et construire son histoire de sujet? Comment pourrais-je faire ce 
travail-là avec lui et l'aider à faire ce travail sur lui-même si mon propre savoir est détaché de mon historicité culturelle? Comment pourrais-je faire ça!? C'est là que le psychotique oblige les thérapeutes à faire un travail de culture. MP : Et ce travail, qui s'inscrit nécessairement au cœur du transfert, ne détermine-t-il pas en quelque sorte votre place comme analyste?

EJ : D’emblée, je suis idéologiquement pris pour quelqu'un qui est le nonreprésentant du west side et ça m'a obligé à faire un travail de culture personnel très important. En somme, à partir de quelle place mon travail de psychanalyste opère-t-il, me demandez-vous, aussi bien avec les ouvriers immigrés en Europe du temps où je travaillais en France, qu'avec les Tunisiens. Quel lien historicisé est-ce que j'établis en tant que Sujet et au présent, entre la place du Savoir implicite à mes référents culturels et le cadre théorique auquel je me réfère? Si je ne me posais pas ces questions, je ne pourrais pas assumer pour l'analysant, le patient ou le groupe, un rôle de support à travers lequel les uns et les autres peuvent découvrir leurs difficultés et les possibilités de se déconstruire, de se construire et de se reconstruire en tant que Sujets. Ils ne pourraient pas porter leur propre discours et leur projet personnalisé et historicisé d'Être-là-dans-le-monde.

Cela est particulièrement évident lorsqu'on travaille dans un contexte de transculturalité et encore davantage avec des patients psychotiques. Un tel travail de culture - chez l'analyste mais aussi chez l'analysant - s'avère incontournable pour qu'un sujet malade de schizophrénie, par exemple, et qui se vit hors temps, puisse progressivement se réapproprier son image du corps et son histoire. Le thérapeute ne saurait se passer d'un travail supplémentaire sur lui-même pour décoder, entre autres, l'articulation endogène entre le savoir théorique et pratique d'aujourd'hui auquel il se réfère, et le savoir implicite dans la dynamique endogène de sa culture. Il s'agit par exemple pour moi de décoder une filiation du savoir, de la psychanalyse, du fondateur de la psychanalyse, avec quelqu'un comme Abraham Aboulafia, le fondateur de la Kabbale. Autrement dit, il s'agit de décoder au présent l'articulation d'une chaîne de filiation au savoir qui remonte jusqu'à Aristote en passant par Ibn Sina: Lacan, Freud et la kabbale de la mystique juive et sa référence à Aboulafia qui fut lui-même l'élève de Moïse Maïmonide, qui lui-même était lié en termes de filiation symbolique, outre son savoir mystique, au savoir médical et philosophique, et donc ouvert à la démarche rationaliste quant à l'approche de la question métaphysique du sens. Sur ce plan, il avait un rapport de filiation directe avec Ibn Rochd (Averroès) qui se reconnaissait luimême pour maîtres Ibn Sina (Avicenne) et Aristote. 
Tout travail psychanalytique implique en définitive un travail de culture aussi bien pour l'analyste que pour l'analysant (en individuel comme en groupe). Mais ce travail de culture ne saurait signifier qu'il y aurait à tenir compte des spécificités culturelles ouvrant à des méthodologies de pratiques différentes selon les cultures, de l'ordre de ce qui est censé relever de l'ethnopsychanalyse, comme si la psychanalyse en tant que science pouvait avoir besoin d'un sous-produit usant de la pensée magique pour des sous-cultures. Un travail de culture se déploie en fait en tant qu'expérience de transculturalité, au prix de moments d'errance, ouvrant l'accès à une nouvelle perspective, à un nouvel éclairage pour donner plus d'épaisseur et de profondeur aux concepts théoriques et méthodologiques. Cela permet de dépasser les risques omniprésents d'aplatir ces concepts dans une compréhension simplificatrice, en surface, et purement digitale. Cela permet de répondre enfin aux vœux de Freud qu'elle puisse transformer et « rendre meilleur le caractère des psychanalystes », de telle sorte qu'ils deviennent plus humanistes et plus attachés à l'éthique.

Cette réflexion sur la psychanalyse comme travail de culture m'a beaucoup aidé à travailler avec les psychotiques. Heureusement qu'ils sont là pour interroger la psychanalyse et lui demander où elle va, de même qu'ils posent cette question à l'ensemble des sciences humaines. Et pour nous obliger à reprendre la question que posait Ernest Jones en 1943 dans un fantastique et très actuel article: How can civilization be saved? Comment, en effet, sauver la civilisation? Actuellement, nous prônons l'idéologie sécuritaire non seulement, pour les malades psychotiques, mais pour le monde entier. Or, ce qui s'est passé en Tunisie, c'est un début de libération de ce carcan sécuritaire. MP: Ce travail de culture implique donc la nécessité de dévoiler le refoulé culturel de l'Occident. C'est dans la mesure où nous pouvons travailler ce refoulé que nous pouvons faire obstacle ou en tout cas problématiser cette dimension qui s'installe de plus en plus fortement, qui imprègne de plus en plus toutes les structures de soins publiques dans les pays occidentaux. EJ : Je vais essayer de reprendre, pour illustrer l'idéologie sécuritaire en passant par une démarche pratique. Quand un malade psychotique est dans sa famille et qu'il rechute sur un mode aigu alors qu'il est stabilisé sur le plan du traitement chimique, on se rend compte que c'est souvent parce qu'il a parlé quelque chose de l'ordre de l'indicible, de l'ordre du refoulé originaire dans le roman familial. Même quand il commence à s'améliorer parce qu'il est suivi en psychothérapie, quelle que soit la technique et l'étayage théorique de cette prise en charge, le patient rechute dans une institution représentée 
par une équipe soignante dont les membres ne sont pas impliqués dans son roman familial. Il arrive également que ce psychotique soit dans l'errance dans le champ social et qu'il se mette à parler de quelque chose qui l'entraîne à un agir violent. Il faut le dire! Nous avons alors un choix: ou nous disons que nous ne pouvons pas écouter ces malades, que nous ne pouvons pas les traiter et qu'il faut donc recourir au sécuritaire; ou nous acceptons qu'ils interrogent notre mode d'écoute et qu'ils interrogent nos difficultés à penser le refoulé culturel qu'ils nous renvoient. Je ne parle même pas ici d'Occident ou d'Orient parce que dans chaque société, il y a un refoulé culturel.

MP : Au terme de notre entretien, on se rend compte - sur les plans théorique et clinique, ainsi qu'à travers toutes ces questions de refoulé culturel et de processus imaginaires - que ce qui devient fondamental, c'est que le psychotique (et pas que le psychotique, mais vous insistez particulièrement sur le psychotique) met en exergue les dangers de dérive vers les idéologies sécuritaires qui imprègnent aujourd'hui... pas de façon globale, mais de façon assez importante, les équipes de soin, les dispositifs publics et l'ensemble de l'industrie médicale. Bref, le psychotique vient là dire quelque chose qui est de l'ordre de l'indicible certes, mais de l'ordre de l'indicible aux niveaux politique, idéologique, sociologique, bref à plusieurs niveaux. Est-ce que vous pourriez nous en dire un peu plus sur ce sujet?

EJ : Ce que vous dites illustre la nécessité que la thérapie soit assumée en tant que travail de culture par les thérapeutes, tout comme par le patient, tout comme par l'analysant d'ailleurs. Les choses sont caricaturales parce que la régression aiguë d'un malade psychotique a lieu au niveau du mécanisme d'effondrement dont nous parlions tout à l'heure. Il sollicite alors une protection qui ne lui a pas été apportée puisqu'on dénie la chose dont il venait de parler. Généralement, en famille, le psychotique, qui était jusque-là bien équilibré, stabilisé, voire assez bien intégré socialement, pouvait même avoir repris une petite activité. Et voilà qu'au cours du suivi il rechute. Parce qu'il a dit une parole de l'ordre de l'impensé, qui ne doit pas se dire parce qu'il s'agit de quelque chose de l'ordre du refoulé dans le roman familial. C'est alors qu'il est hospitalisé d'urgence. Tout le monde s'entend pour dire qu'il y a un rapport entre la psychose, les rechutes psychotiques et les perturbations de la structure familiale. La rechute n'a jamais lieu comme ça, spontanément. Elle a eu lieu en réaction à une parole qui est de l'ordre de l'indicible, qui a été perçue comme telle alors que l'équipe soignante a participé à la contenir. Cette équipe est formée par des représentants des différentes catégories sociales, des différentes catégories des fonctions de soi et des différentes 
catégories d'âges, incluant la famille du malade. Le malade a donc parlé quelque chose qui n'est pas seulement de l'ordre du refoulé familial, mais qui est également de l'ordre du refoulé culturel. Et c'est ça que j'ai développé dans Filiation et altérité. Il y a à ce moment-là obligation pour les thérapeutes de travailler en équipe, de faire ce travail de culture qui oblige à repenser quelle a été la difficulté à entendre ce discours et ce qu'il y a derrière cette difficulté. Quand nous travaillions ensemble en séminaire, nous effectuions un travail de culture extraordinaire. Ce travail ne peut se faire qu'en groupe et quand il se fait, les malades ensuite se sentent beaucoup mieux. D'ailleurs, ils sentent que ça apporte beaucoup, non seulement au patient pour lequel on a étudié la difficulté, mais même pour les autres patients. Ils ressentent donc de l'amélioration. Quand l'équipe soignante en arrive à dire par exemple: «Tel sujet, non, on arrive pas à en parler ", c'est qu'on respecte la résistance de l'équipe. On se dit alors: bon, on va circonscrire cette case vide, ça va représenter une case vide dont on ne parlera pas, mais on va parler à la périphérie de cette case et de la périphérie, on va délimiter cet espace. Quand on fait un travail de culture, on n'est pas là pour forcer des gens à abattre les résistances. Les résistances constituent un mécanisme positif, mais à condition d'en parler et de dire là où nous ne pouvons pas en parler, de circonscrire cet espace et de le respecter. MP: C'est ce qui empêche d'être pris par les idéologies sécuritaires.

EJ: Oui! Alors, si on ne fait pas ce travail de culture, bien sûr on reste dans le confort. Nous n'allons pas nous en vouloir, la famille non plus, qui est d'ailleurs épuisée. Tout le monde dira «C'est vrai: le psychotique, le pauvre, on ne peut rien faire pour lui. Vous avez raison, vous avez fait ce que vous pouvez, vous ne pouvez rien pour lui. » Et on laisse place à ce moment-là au sécuritaire pour protéger la société et pour protéger la famille.

MP: Vous rejoignez Foucault qui disait qu'il faut protéger la société.

EJ : Voilà. Et je trouve que c'est là que nous devrions réfléchir à la question de Jones parce qu'à travers ça, le sécuritaire pour le malade mental, il y a tout le sécuritaire pour la migration, tout le sécuritaire pour les échanges, le sécuritaire à l'intérieur de n'importe quelle équipe pour les échanges de savoir, la compartimentalisation du savoir. Il ne faut pas croire que le sécuritaire ne touche que les pays émergents, il touche également la démocratie dans les pays développés. En tout cas, le malade psychotique, dans tous les pays du monde, pose le problème d'une certaine organisation sociale au sens d'une déshumanisation et de ce que Milner a récemment appelé l'évolution vers la république des choses, où plus personne n'est sujet de son savoir, n'est sujet de son agir, chacun devenant en principe autonome et indépendant comme 
individu, mais cela dans la pratique ultra-dépendante de l'organisation et du management.

MP: Essedik Jeddi, je vous remercie beaucoup. C'était passionnant.

\section{Note}

1. Groupe interuniversitaire de recherche en anthropologie médicale et en ethnopsychiatrie.

\section{Principales publications d'Essedik Jeddi}

1968, Influence de l'anesthésie sur l'action thermogène des catécholamines au cours de l'adaptation au froid. Thèse de médecine, Lyon, Faculté de médecine.

1970, Confort du contact et thermorégulation comportementale, Physiology \& Behavior, V, 1487-1493.

1971, En collaboration avec M. Beaujard, Syndrome thermorégulateur subjectif réactionnel à l'insatisfaction du besoin de maternage dans la psychose infantile, Journal médical de Lyon, 1, 207, 659-673.

1971, Approche phénoménologique de la criminalité et de l'intentionnalité dite criminelle à partir des facteurs sociogènes des processus de psychotisation chez l'ouvrier migrant, Rapport de réunion de l'Association Médecine Légale et Criminologie, Palais de Justice de Lyon. Publié en partie in Instantanés criminologiques, 17-26.

1972,Aspect sociogène de la morbidité psychiatrique chez l'ouvrier arabe transplanté en France, Comptes rendus du Congrès de l'Association de psychiatrie et de neurolologie de langue française (Tunis), Paris, Masson, 1407-1420.

1972, Thermoregulatory Efficiency of Neonatal Rabbit Search for Fur Comfort Contact, International Journal of Biometeorology, 15 (2-4), 337-341.

1977, Ontogenèse du confort thermique et hypothèses théorique du facteur thermotactile dans le développement affectif, in J. Durant et J. Raynaud (éds.), Confort thermique: aspects physiologiques et psychologiques, Paris, INSERM, 101-144.

1981, Cuerpo y cultura, Clinic y analyses grupal, revista de psychothérapia y psychologica social aplicada (Madrid), 37, 678-700.

1981, Le corps en psychiatrie, Paris, Masson.

1985, Aspects culturels de l'évènement, in J. Guyotat et P. Fedida (éds.), Symposium Événement et Psychopathologie, Lyon, SIMEP.

1985, Paradoxes en miroir et signes, International Journal of Psychology, 20, 449-470.

1985, Psychose, Famille et Culture, Paris, L'Harmattan.

2001, The Bodily Interface Between Biological and Cultural-Symbolic Organizers (from Symbiosis to individuation), in Marco. M. Olivetta (éd.), Incarnation, Rome, Bibliotheca dell'Archivio di Filosofia - CEDAM, 337-350.

2004, De la prise en charge au long cours des malades psychotiques chroniques au Maroc et ses aléas et Proposition d'un projet d'une expérience pilote dans un Centre de Jour à Casablanca et un Centre d'Hébergement à Rabat pour malades mentaux chroniques, Rapport OMS en 2 parties.

2010, Filiation et altérité. Psychanalyse et malaise dans la psychiatrie, Montréal, Liber.

2012, Les idéologies sécuritaires, Montréal, Mémoire d'encrier (introduction de Michel Peterson).

2012, Psychothérapie institutionnelle et musicothérapie, Tunis, Simpact (avant-propos de Michel Peterson; Présentation de Youssef Seddik). Livre accompagné d'un DVD du film El-Baab (la porte), réalisé par Essedik Jeddi, 27 minutes. 


\section{Filmographie}

1980, Al-Baab/La Porte ou 'Art-thérapie et institution', en collaboration avec M. Lefayef, C. Mili,

A. Bouzgaroru et le collectif de l'Unité IBN ROCHD - Pinel, Film 52 min, 16 mm couleur, et $27 \mathrm{~min} 16 \mathrm{~mm}$ couleur, version arabe, française et anglaise. Diffusion R.T.T (Tunisie).

1996, Al-Mâwna, Film documentaire vidéo, 26min, réalisé avec Salem BEN AMOR.

2002, El-Loud. Approche anthropologique, Film $44 \mathrm{~min}$, versions arabe, française, et sous-titrée en anglais. Production: Razi Films. 